\title{
Solution of Dirac Equation with Modified Hylleraas Potential under Spin and Pseudospin Symmetry
}

\author{
Akpan N.Ikot ${ }^{1, *}$, E. Maghsoodi ${ }^{2}$, Oladunjoye O.Awoga ${ }^{1}$, S. Zarrinkamar ${ }^{3}$ and H. Hassanabadi ${ }^{2}$, \\ ${ }^{1}$ Theoretical Physics Group, Department of Physics,University of Uyo-Nigeria, Nigeria \\ ${ }^{2}$ Department of Basic Sciences, Shahrood Branch, Islamic Azad University, Shahrood, Iran \\ ${ }^{3}$ Department of Basic Sciences, Garmsar Branch, Islamic Azad University, Garmsar, Iran
}

Received: 12 Sep. 2013, Revised: 20 Jan. 2014, Accepted: 25 Jan. 2014

Published online: 1 Apr. 2014

\begin{abstract}
We obtain the bound energy spectrum and the corresponding generalized hypergeometric wave functions of the Dirac equation for modified-Hylleraas potential under spin and pseudospin symmetry limits within the framework of the Alhaidari-formalism. This is accomplished by approximating the spin-orbital term in the Dirac equation rather than the orbital term in the resulting Schr?dinger-like equation using the modified parametric generalization of the Nikiforovmethod.
\end{abstract}

Keywords: Alhaidari-formalism, Dirac equation Hylleraas-potential,Nikiforov-Uvarov method.

\section{Introduction}

Within the frame work of the relativistic Dirac equation, the concepts of exactpseudospin symmetry occurs when the Lorentz scalar potential $S(r)$ and the vector potential $V(r)$ are equal but opposite in sign i.e. $S(r)=-V(r)$. However, approximate pseudo spin symmetry is when the sum of the potential is $\Sigma(r)=S(r)+V(r)=C_{p s}=$ Cons. $\neq 0 \quad[1,2] . \quad$ The pseudospinsymmetry is used to establish effective shell model [3]. On the other hand, exact spin symmetry arises if thescalar potential $S(r)$ and vector $V(r)$ are equal i.e $S(r)=V(r) \quad$.However, in nuclei the difference potential $\Delta(r)=V(r)-S(r)=C_{s}=$ Cons. $\neq 0 \quad$ [4]. The spin symmetry is relevant in meson [5]. Recently, many authors have investigated the Dirac equation approximately with spin and pseudospin symmetries for different potential models such as the Hulthen potential [6], generalized Morse potential [7], the Dirac-Hulthen problem [8], Hulthen potential including Coulomb-like tensor potential [9], the Woods-Saxon [10], Relativistic Morse potential [11] and others [12]. The bound state solution of the Dirac equation under spin and pseudospin symmetries have been obtain using various methods such as the supersymmetric quantum mechanics (SUSY) [13], the Nikiforov-Uvarov method [14] and others [15]. Ikot [16]has investigated the Dirac equation with Hyperbolical potential including Coulomb-like tensor potential under spin symmetry for any spin-orbit and the wave functions and the corresponding energy eigenvalue have been calculated using the improved approximation scheme for the centrifugal term $\frac{\kappa(\kappa \pm 1)}{r^{2}}$. Different authors have found the bound state energy spectrum of some physical quantities whose behaviour depends on the behaviour of the system near the singularity [17]. The energy spectrum for instance, depends strongly on the angular momentum resulting from $\frac{1}{r^{2}}$ singularity of the orbital term even for high excited states [17]. However, because $\frac{1}{r^{2}}$ is too singular, the validity of such approximation is limited to very few of the lowest energy states. In order to extend the approximation to higher energy states, Alhaidari [18] for the first time evaluated the Dirac equation with coupling to $\frac{1}{r}$ singular potential for all angular momenta.Now, the solution of Dirac equation with Hylleraas potential is very difficult to evaluate because of the nature of its potential,$V(r)=\frac{V_{0}\left(a+e^{\lambda r}\right)}{\left(b+e^{\lambda r}\right)}$, where $a, b$ are the Hylleraas parameters with $a \neq b$ Moreover, this potential is singular at $r=r_{0}$, where $b=-e^{\lambda r_{0}}$, and the singularity is $r^{-1}$ type which is compatible with Alhaidari's approach. Nevertheless, with the formalism of Dirac equation proposed by Alhaidari [17] and the generalized parametric Nikiforov-Uvarovmethod, we

\footnotetext{
*Corresponding author e-mail: ndemikotphysics@gmail.com, h.hassanabadi@ shahroodu.ac.ir
} 
attempt to find analytical approximate solution of Dirac equation with Hylleraas potential including the energy spectrum and the corresponding wave functions within spin and pseudospin limits. Since Hylleraas proposed this potential [25] no much work has been reported on the bound state solution. Recently, we first attempt to report on the bound state solution of this potential [26]. In this paper, we are motivated by this success and attempt to solve the modified Hylleraas potential under spin and pseudospin symmetry using the parametric NU method. The results obtained in this work when $b \approx-1$, or equivalently, $r_{0}=0$ is more accurate over a wider range as claimed by Alhaidari [17] The organization of the paper is as follows. In section 2, we review the NU method. Bound state solution of Dirac equation is presented in section 3.Finally; we give a brief conclusion in section 4.

\section{Parametric Generalization of Nikiforov-Uvarov Method}

The concept of NU method [14] was proposed to solve the second-order linear differential equation by reducing it to a generalized equation of hypergeometric-type of the form

$$
\frac{d^{2} \Psi(s)}{d s^{2}}+\frac{\tilde{\tau}(s)}{\sigma(s)} \frac{d \Psi(s)}{d s}+\frac{\tilde{\sigma}(s)}{\sigma^{2}(s)} \Psi(s)=0,
$$

where the prime denote the differential with respect to $s, \sigma(s), \tilde{\sigma}(s)$ are polynomials at most second degree and $\tilde{\tau}(s)$ is the first-degree polynomials. The solution of Eq. (1) is obtain by using a common ansatz for the wave function as

$$
\Psi(s)=\varphi(s) \chi_{n}(s)
$$

which reduces Eq. (2) into a hypergeometric-type equation,

$$
\sigma(s) \frac{d^{2} \chi_{n}(s)}{d s^{2}}+\tau(s) \frac{d \chi_{n}(s)}{d s}+\lambda \chi_{n}(s)=0,
$$

where $\varphi(s)$ is defined as a logarithmic derivative [14]

$$
\frac{\varphi^{\prime}(s)}{\varphi(s)}=\frac{\pi(s)}{\sigma(s)}
$$

we consider $\pi(s)$ and $\tau(s)$ for the NU method as

$$
\begin{gathered}
\pi(s)=\frac{\sigma^{\prime}-\tilde{\tau}}{2} \pm \sqrt{\left(\frac{\sigma^{\prime}-\tilde{\tau}}{2}\right)^{2}-\tilde{\sigma}(s)+k \sigma(s),} \\
\tau(s)=\tilde{\tau}(s)+2 \pi(s),
\end{gathered}
$$

and the other wave function is the hypergeometric type function whose polynomial solution satisfies the Rodriques relation

$$
\chi_{n}(s)=\frac{B_{n}}{\rho(s)} \frac{d^{n}}{d s^{n}}\left[\sigma^{n}(s) \rho(s)\right],
$$

where $B_{n}$ is the normalization constant and the weight function $\rho(s)$ satisfy the condition

$$
(\sigma(s) \rho(s))^{\prime}=\tau(s) \rho(s),
$$

The required $\lambda$ for the NU method are defined as

$$
\lambda=k+\pi^{\prime}(s)
$$

Thus, the determination of $k$ in Eq. (5) is the necessary step in the calculation of $\pi(s)$ for which the discriminant of the square root in Eq. (5) is set to zero. The new eigenvalues equation now takes the form

$$
\lambda=\lambda_{n}=-n \tau^{\prime}(s)-\frac{n(n-1) \frac{d^{2} \sigma(s)}{d s^{2}}}{2}, n=0,1,2
$$

and its derivative is negative which is the necessary condition for bound state solutions. The energy eigenvalue is obtained by comparing Eqs. (9) and (10). The parametric generalization of the NU method that is valid for both central and non-central exponential-type potential has been proposed [19]. We use the parametric generalization of the NU method as

$\frac{d^{2} \Psi(s)}{d s^{2}}+\frac{\alpha_{1}-\alpha_{2} s}{s\left(1-\alpha_{3} s\right)} \frac{d \Psi(s)}{d s}+\frac{1}{s^{2}\left(1-\alpha_{3} s\right)^{2}}\left[-\xi_{1} s^{2}+\xi_{2} s-\xi_{3}\right] \Psi(s)=0$,

Now comparing Eq. (11) and Eq. (1), we obtain the following parametric polynomials

$$
\begin{gathered}
\tilde{\tau}(s)=\alpha_{1}-\alpha_{2} s, \\
\sigma(s)=s\left(1-\alpha_{3} s\right), \\
\tilde{\sigma}(s)=-\xi_{1} s^{2}+\xi_{2} s-\xi_{3},
\end{gathered}
$$

Substituting Eqs. (12 - 14) into Eq. (7), we find

$$
\pi(s)=\alpha_{4}+\alpha_{5} s \pm\left[\left(\alpha_{6}-\alpha_{3} k_{ \pm}\right) s^{2}+\left(\alpha_{7}+k_{ \pm}\right) s+\alpha_{8}\right]^{1 / 2},
$$

where

$\alpha_{4}=\frac{1}{2}\left(1-\alpha_{1}\right), \alpha_{5}=\frac{1}{2}\left(\alpha_{2}-2 \alpha_{3}\right), \alpha_{6}=\alpha_{5}^{2}+\xi_{1}, \alpha_{7}=2 \alpha_{4} \alpha_{5}-\xi_{2}, \alpha_{8}=\alpha_{4}^{2}+\xi_{3}$,

We obtain the parametric $k_{ \pm}$from the condition that the function under the square root should be square of a polynomial

$$
k_{ \pm}=-\left(\alpha_{7}+2 \alpha_{3} \alpha_{8}\right) \pm 2 \sqrt{\alpha_{8} \alpha_{9}},
$$

where

$$
\alpha_{9}=\alpha_{3} \alpha_{7}+\alpha_{3}^{2} \alpha_{8}+\alpha_{6},
$$

Hence, the $\pi(s)$ in Eq. (15) becomes

$$
\pi(s)=\alpha_{4}+\alpha_{5} s-\left[\left(\sqrt{\alpha_{9}}+\alpha_{3} \sqrt{\alpha_{8}}\right) s-\sqrt{\alpha_{8}}\right],
$$

for the negative $k_{-}$values

$$
k_{-}=-\left(\alpha_{7}+2 \alpha_{3} \alpha_{8}\right)-2 \sqrt{\alpha_{8} \alpha_{9}},
$$


Thus, from the relation, $\tau(s)=\tilde{\tau}(s)+2 \pi(s)$, we have

$\tau(s)=\alpha_{1}+2 \alpha_{4}-\left(\alpha_{2}-2 \alpha_{5}\right) s-2\left[\left(\sqrt{\alpha_{9}}+\alpha_{3} \sqrt{\alpha_{8}}\right) s-\sqrt{\alpha_{8}}\right]$,

whose derivative must be negative beginequation

$$
\tau^{\prime}(s)=-2 \alpha_{3}-2\left(\sqrt{\alpha_{9}}+\alpha_{3} \sqrt{\alpha_{8}}\right)<0,
$$

Solving Eqs. (8) and (9), we obtain the parametric energy equation as

$\alpha_{2} n+n(n-1) \alpha_{3}-(2 n+1) \alpha_{5}+(2 n+1)\left[\sqrt{\alpha_{9}}+\alpha_{3} \sqrt{\alpha_{8}}\right]+\alpha_{7}+2 \alpha_{3} \alpha_{8}+2 \sqrt{\alpha_{8} \alpha_{9}}=0$,

The weight function $\rho(s)$ is obtain as

$$
\rho(s)=s^{\alpha_{10}-1}\left(1-\alpha_{3} s\right)^{\frac{\alpha_{11}}{\alpha_{3}}-\alpha_{10}-1},
$$

and together with Eq. (9), we obtain

$$
\chi_{n}(s)=P_{n}^{\left(\alpha_{10}-1, \frac{\alpha_{11}}{\alpha_{3}}-\alpha_{10}-1\right)}\left(1-2 \alpha_{3} s\right),
$$

where

$$
\begin{gathered}
\alpha_{10}=\alpha_{1}+2 \alpha_{4}+2 \sqrt{\alpha_{8}} \\
\alpha_{11}=\alpha_{2}-2 \alpha_{5}+2\left(\sqrt{\alpha_{9}}+\alpha_{3} \sqrt{\alpha_{8}}\right),
\end{gathered}
$$

and $P_{n}^{(\alpha, \beta)}(s)$ are the Jacobi polynomials. The other part of the wave function is obtained from Eq. (4) as

$$
\varphi(s)=s^{\alpha_{12}}\left(1-\alpha_{3} s\right)^{-\alpha_{12}-\frac{\alpha_{13}}{\alpha_{3}}}
$$

where

$$
\begin{gathered}
\alpha_{12}=\alpha_{4}+\sqrt{\alpha_{8}}, \\
\alpha_{13}=\alpha_{5}-\left(\sqrt{\alpha_{9}}+\alpha_{3} \sqrt{\alpha_{8}}\right),
\end{gathered}
$$

Thus, the total wave function becomes

$\Psi(s)=N_{n} s^{\alpha_{12}}\left(1-\alpha_{3} s\right)^{-\alpha_{12}-\frac{\alpha_{13}}{\alpha_{3}}} P_{n}^{\left(\alpha_{10}-1, \frac{\alpha_{11}}{\alpha_{3}}-\alpha_{10}-1\right)}\left(1-2 \alpha_{3} s\right)$,

where $N_{n}$ is the normalization constant.

\section{Formulation of the problem}

The Dirac equation of a single nucleon of rest mass $M$ scalar potential $S(r)$ and vector potential $V(r)$ (in the relativistic unit $(\hbar=c=1)$ is [20]

$$
[\alpha \cdot p+\beta(M+S(r))] \Psi(r)=[E-V(r)] \Psi(r),
$$

where $E$ is the relativistic energy of the system and $p=$ $-i \nabla$ is the three dimensional momentum operator, $\alpha$ and $\beta$ are the $4 \times 4$ Dirac matrices defined as

$$
\alpha=\left(\begin{array}{ll}
0 & \sigma \\
\sigma & 0
\end{array}\right), \beta=\left(\begin{array}{cc}
I & 0 \\
0 & -I
\end{array}\right)
$$

where $\sigma$ is the Pauli matrices and $I$ is the $2 \times 2$ unitary matrix. The total angular momentum operator $J$ and spin orbit $K=(\sigma . L+1)$, where is orbital angular nucleon commute with Dirac Hamiltonian. The eigenvalues of spin-orbit coupling operator are $K=\left(j+\frac{1}{2}\right)>0$ and $K=-\left(j+\frac{1}{2}\right)<0$ for unaligned spin $j=l-\frac{1}{2}$ and the aligned spin $j=l+\frac{1}{2}$ respectively. The complete set of the conservative quantities are denoted as $(n, \kappa, j, l, m)$ Thus, the Dirac spinors can be written according to the radial quantum number and spin-orbit coupling number as follows:

$$
\Psi_{n \kappa}(r)=\frac{1}{r}\left(\begin{array}{cc}
F_{n \kappa}(r) & Y_{j m}^{l}(\theta, \varphi) \\
i G_{n \kappa}(r) & Y_{j m}^{\tilde{l}}(\theta, \varphi)
\end{array}\right)
$$

where $F_{n \kappa}(r)$ is the upper component and $G_{n \kappa}(r)$ is the lower component of the Dirac spinors and $m$ is the projection of the angular momentum on the z-axis and $l(l+1)=\kappa(\kappa+1), \tilde{l}(\tilde{l}+1)=\kappa(\kappa-1)$. On substituting Eqs. (33)and (34) into Eq. (32), we obtain two coupled deferential equations for the upper and the lower radial wave function $F_{n \kappa}(r)$ and $G_{n \kappa}(r)$ as

$$
\begin{aligned}
& \left(\frac{d}{d r}-\frac{\kappa}{r}\right) G_{n \kappa}(r)=\left(M-E_{n \kappa}+\Sigma(r)\right) F_{n \kappa}(r), \\
& \left(\frac{d}{d r}+\frac{\kappa}{r}\right) F_{n \kappa}(r)=\left(M+E_{n \kappa}-\Delta(r)\right) G_{n \kappa}(r),
\end{aligned}
$$

where

$$
\begin{aligned}
& \Delta(x)=V(x)-S(x), \\
& \Sigma(x)=V(x)+S(x),
\end{aligned}
$$

Eliminating $F_{n \kappa}(r)$ and $G_{n \kappa}(r)$ from Eq. (35) and (36), we obtain the following second Schr?dinger-like differential equations for the upper and lower components of the Dirac wave equation as

$\left\{\left(\frac{d^{2}}{d r^{2}}-\frac{\kappa(\kappa+1)}{r^{2}}\right)-\left[M+E_{n \kappa}-\Delta(r)\right]\left[M-E_{n \kappa}+\Sigma(r)\right]+\frac{\frac{d \Delta(r)}{d r}\left(\frac{d}{d r}-\frac{\kappa}{r}\right)}{\left[M+E_{n \kappa}-\Delta(r)\right]}\right\} F_{n \kappa}(r)=0$,

$\left\{\left(\frac{d^{2}}{d r^{2}}-\frac{\kappa(\kappa-1)}{r^{2}}\right)-\left[M+E_{n \kappa}-\Delta(r)\right]\left[M-E_{n \kappa}+\Sigma(r)\right]+\frac{\frac{d \Sigma(r)}{d r}\left(\frac{d}{d r}-\frac{\kappa}{r}\right)}{\left[M-E_{n \kappa}+\Sigma(r)\right]}\right\} G_{n \kappa}(r)=0$,

when $\Sigma(r)=0$ (pseudospin symmetry) with $E_{n \kappa} \neq M$ and when $\Delta(r)=0$ (spin symmetry) with $E_{n \kappa} \neq-M$. Equations (39) and (40) are the Schrodinger-like equation with coupling to the $\frac{1}{r^{2}}$ singular term satisfying $F_{n \kappa}(r)$ and $G_{n \kappa}(r)$ respectively. For spin symmetry $\frac{d \Delta(r)}{d r}=0$,i.e, $\Delta(r)=C_{s}=$ const. , Eq. (37) turns into

$\left\{\left(\frac{d^{2}}{d r^{2}}-\frac{\kappa(\kappa+1)}{r^{2}}\right)-\left(M+E_{n \kappa}-C_{s}\right) \Sigma(r)+E_{n \kappa}^{2}-M^{2}+C_{s}\left(M-E_{n \kappa}\right)\right\} F_{n \kappa}(r)=0$,

Similarly, the pseudospin symmetry requires $\frac{d \Sigma(r)}{d r}=0$ ,i.e, $\Sigma(r)=C_{p s}=$ const. , Eq. (40) becomes

$\left\{\left(\frac{d^{2}}{d r^{2}}-\frac{\kappa(\kappa-1)}{r^{2}}\right)+\left(M-E_{n \kappa}+C_{p s}\right) \Delta(r)+E_{n \kappa}^{2}-M^{2}-C_{p s}\left(M+E_{n \kappa}\right)\right\} G_{n \kappa}(r)=0$, 
The Schrodinger-like equation of Eqs (41) and (42) coupling with $\frac{1}{r^{2}}$ singular orbit term under spin and pseudospin symmetries have been studied extensively in recent times [21]. However, the limitation of this equation is that the $r^{-2}$ orbital term is too singular and the validity of such approximation is limited only to very few of the lowest energy eigenstates [17]. In order to extend the approximation to a wider energy spectrum, Alhaidari [18] approximate the less singular term distribution $\frac{1}{r}$ in the first order Dirac equation (35) and (36). This approximation of the $\frac{1}{r}$ orbital term in the first order differential equation is less singular since it goes like $\frac{1}{r}$ and not like $\frac{1}{r^{2}}$. Now approximating the $\frac{1}{r}$ spin-orbit term in the Dirac equation of Eqs. (35) and (41) by a singular function $W(r)$ i.e. $W(r) \approx \frac{1}{r}$ resultsin the following second-order differential equations for spin and pseudospin as [17]

$$
\begin{gathered}
\left\{\frac{d^{2}}{d r^{2}}-\kappa^{2} W^{2}(r)+\kappa \frac{d W(r)}{d r}-\left(M+E_{n \kappa}-C_{s}\right) V(r)+E_{n \kappa}^{2}\right. \\
\left.-M^{2}+C_{s}\left(M-E_{n \kappa}\right)\right\} F_{n \kappa}(r)=0
\end{gathered}
$$

and

$$
\begin{gathered}
\left\{\frac{d^{2}}{d r^{2}}-\kappa^{2} W^{2}(r)-\kappa \frac{d W(r)}{d r}+\left(M-E_{n \kappa}+C_{p s}\right) V(r)+E_{n \kappa}^{2}\right. \\
\left.-M^{2}-C_{p s}\left(M+E_{n \kappa}\right)\right\} G_{n \kappa}(r)=0
\end{gathered}
$$

respectively. The proper approximation for $r^{-2}$ is not simply $W^{2}(r)$ but it also involves the derivative $\frac{d W(r)}{d r}$ which gives the supersymmetric form $W^{2}(r) \pm W^{\prime}(r)$. The proper approximation was introduced recently by Alhaidari [18, 22] as

$$
\frac{\kappa(\kappa \pm 1)}{r^{2}}=\kappa^{2} W^{2}(r) \mp \kappa W^{\prime}(r)
$$

In this study we consider $W(r)=\frac{\eta e^{\eta r}}{\left(b+e^{\eta r}\right)}$ where $b=-1$ which is a modified form of the Hylleraas potential under investigation.

\section{Results and Discussion}

\subsection{Spin symmetry Limit}

The spin symmetry arises from the condition $\frac{d \Delta(r)}{d r}=0$ or $\Delta(r)=C_{s}=$ const. [22] then Eq. (9) becomes

$$
\begin{gathered}
\left\{\frac{d^{2}}{d r^{2}}-\frac{\kappa^{2} \eta^{2} e^{2 \eta r}}{\left(e^{\eta r}-1\right)^{2}}-\frac{\kappa \eta^{2} e^{\eta r}}{\left(e^{\eta r}-1\right)^{2}}-\frac{V_{0}\left(M+E_{n \kappa}-C_{s}\right)\left(a+e^{\eta r}\right)}{\left(e^{\eta r}-1\right)}\right. \\
\left.+E_{n \kappa}^{2}-M^{2}+C_{s}\left(M-E_{n \kappa}\right)\right\} F_{n \kappa}(r)=0,
\end{gathered}
$$

We have taken $V(r)=\frac{V_{0}\left(a+e^{\eta r}\right)}{\left(e^{\eta r}-1\right)}$ as the modified Hylleraas potential. If we define the new variable $s=e^{\eta r}-1$, then the positive energy Schrödinger-like equation of Eq. (46) takes the form

$$
\frac{d^{2} F_{n \kappa}(s)}{d s^{2}}+\frac{s}{s(s+1)} \frac{d F_{n \kappa}(s)}{d s}+\frac{1}{s^{2}(s+1)^{2}}\left[-\left(A^{s}-\varepsilon^{2}\right) s^{2}+B^{s} s-C^{s}\right] F_{n \kappa}(s)=0,
$$

where

$$
\begin{gathered}
A^{s}=\kappa^{2}+\beta^{2} \\
B^{s}=-2 \kappa^{2}-\kappa-\beta^{2}(a+1) \\
C^{s}=\kappa[\kappa+1], \varepsilon^{2}=\left[\frac{E_{n \kappa}^{2}-M^{2}+C_{s}\left(M-E_{n \kappa}\right)}{\eta^{2}}\right], \\
\beta^{2}=\frac{V_{0}}{\eta^{2}}\left[E_{n \kappa}+M-C_{s}\right]
\end{gathered}
$$

Comparing Eq. (47) with Eq. (9), we obtain

$$
\begin{gathered}
\alpha_{1}=0, \xi_{1}=A^{s}-\varepsilon^{2}, \\
\alpha_{2}=-1, \xi_{2}=B^{s}, \\
\alpha_{3}=-1, \xi_{3}=C^{s},
\end{gathered}
$$

and from Eqs. (16), (18), (26 - 27) and (29 - 30), we further obtain

$$
\begin{gathered}
\alpha_{4}=\frac{1}{2}, \alpha_{5}=\frac{1}{2}, \alpha_{6}=\frac{1}{4}+A^{s}-\varepsilon^{2} \\
\alpha_{7}=\frac{1}{2}-B^{s}, \alpha_{8}=\frac{1}{4}+C^{s}, \alpha_{9}=A^{s}+B^{s}+C^{s}-\varepsilon^{2}, \\
\alpha_{10}=1+2 \sqrt{\frac{1}{4}+C^{s}}, \alpha_{11}=-2+2\left(\sqrt{A^{s}+B^{s}+C^{s}-\varepsilon^{2}}-\sqrt{\frac{1}{4}+C^{s}}\right), \\
\alpha_{12}=\frac{1}{2}+\sqrt{\frac{1}{4}+C^{s}}, \alpha_{13}=\frac{1}{2}-\left(\sqrt{A^{s}+B^{s}+C^{s}-\varepsilon^{2}}-\sqrt{\frac{1}{4}+C^{s}}\right) .
\end{gathered}
$$

In addition, the energy eigenvalue equation can be obtained from Eq. (21) as

$$
\begin{aligned}
& -n^{2}-n+(2 n+1)\left(\sqrt{A^{s}+B^{s}+C^{s}-\varepsilon^{2}}-\sqrt{\frac{1}{4}+C^{s}}\right) \\
& -B^{s}-2\left(\frac{1}{4}+C^{s}\right)+2 \sqrt{\left(\frac{1}{4}+C^{s}\right)\left(A^{s}+B^{s}+C^{s}-\varepsilon^{2}\right)}=0,
\end{aligned}
$$

By substituting the explicit values of $\beta^{2}, \varepsilon^{2}, A^{s}, B^{s}$ and $C^{s}$, we obtain the energy spectrum for the deformed Hylleraas potential in the Dirac theory for the spin symmetry limit case as

$$
-n^{2}-n+(2 n+1)\left(\sqrt{\frac{-V_{0} a}{\eta^{2}}\left[E_{n \kappa}+M-C_{s}\right]-\left[\frac{E_{n \kappa}^{2}-M^{2}+C_{s}\left(M-E_{n \kappa}\right)}{\eta^{2}}\right]}\right.
$$

$$
\begin{gathered}
\left.-\sqrt{\frac{1}{4}+\kappa[\kappa+1}\right]-\kappa+\frac{V_{0}}{\eta^{2}}\left[E_{n \kappa}+M-C_{s}\right](a+1)-\frac{1}{2} \\
+2 \sqrt{\left(\frac{1}{4}+\kappa[\kappa+1]\right)\left(-\frac{V_{0} a}{\eta^{2}}\left[E_{n \kappa}+M-C_{s}\right]-\left[\frac{E_{n \kappa}^{2}-M^{2}+C_{s}\left(M-E_{n \kappa}\right)}{\eta^{2}}\right]\right)}=0,
\end{gathered}
$$

On the other hand, to find the corresponding wave function, we find from Eq. (31), the functions

$$
\begin{gathered}
F_{n \kappa}(r)=N_{n \kappa}\left(e^{\eta r}-1\right)^{\frac{1}{2}+\sqrt{\frac{1}{4}+\kappa[\kappa+1]}} \\
\left(e^{\eta r}\right)^{-} \sqrt{-\frac{V_{0} a}{\eta^{2}}\left[E_{n \kappa}+M-C_{S}\right]-\left[\frac{E_{n \kappa}^{2}-M^{2}+C_{S}\left(M-E_{n \kappa}\right)}{\eta^{2}}\right]}
\end{gathered}
$$




$$
\begin{gathered}
{ }_{2} F_{1}\left(-n, n+2 \sqrt{\frac{1}{4}+\kappa[\kappa+1}\right] \\
-2 \sqrt{-\frac{V_{0} a}{\eta^{2}}\left[E_{n \kappa}+M-C_{s}\right]-\left[\frac{E_{n \kappa}^{2}-M^{2}+C_{s}\left(M-E_{n \kappa}\right)}{\eta^{2}}\right]}+1 \\
\left.2 \sqrt{\frac{1}{4}+\kappa[\kappa+1]}+1 ; 1-e^{\eta r}\right)
\end{gathered}
$$

Finally, the lower spinor components of the Dirac equation can be obtained from Eq. (36) as

$$
G_{n \kappa}(r)=\frac{1}{\left(E_{n \kappa}+M-C_{S}\right)}\left[\frac{d}{d r}+\kappa W(r)\right] F_{n \kappa}(r),
$$

where $E_{n \kappa} \neq-M+C_{s}$ and only positive energy solutions are valid [11]

\subsection{Pseudospin symmetry limit}

The Dirac equation for pseudospin symmetry could be found from equation (44). If we make a change of variables $s=b+e^{\eta r}$ where $b=-1$, we can rewrite Eq. (42) as

$$
\begin{gathered}
\frac{d^{2} G_{n \kappa}(s)}{d s^{2}}+\frac{s}{s(s+1)} \frac{d G_{n \kappa}(s)}{d s}+\frac{1}{s^{2}(s+1)^{2}}\left[-\left(A^{p s}-\varepsilon^{2}\right) s^{2}\right. \\
\left.+B^{p s} s-C^{p s}\right] G_{n \kappa}(s)=0
\end{gathered}
$$

where

$$
\begin{gathered}
A^{p s}=\kappa^{2}-\beta^{2} \\
B^{p s}=-2 \kappa^{2}+\kappa+\beta^{2}(a+1) \\
C^{p s}=\kappa[\kappa-1], \varepsilon^{2}=\left[\frac{E_{n \kappa}^{2}-M^{2}-C_{p s}\left(M+E_{n \kappa}\right)}{\eta^{2}}\right] \\
\beta^{2}=\frac{V_{0}}{\eta^{2}}\left[M-E_{n \kappa}+C_{p s}\right]
\end{gathered}
$$

The energy equation for the pseudospin limit can be obtain as

$$
\begin{gathered}
-n^{2}-n+(2 n+1) \\
\left.\sqrt{\frac{V_{0} a}{\eta^{2}}\left[M-E_{n \kappa}+C_{p s}\right]-\left[\frac{E_{n \kappa}^{2}-M^{2}-C_{p s}\left(M+E_{n \kappa}\right)}{\eta^{2}}\right]}-\sqrt{\frac{1}{4}+\kappa[\kappa-1]}\right) \\
+\kappa-\frac{V_{0}}{\eta^{2}}\left[M-E_{n \kappa}+C_{p s}\right](a+1)-\frac{1}{2} \\
+2 \sqrt{\left(\frac{1}{4}+\kappa[\kappa-1]\right)\left(\frac{V_{0} a}{\eta^{2}}\left[M-E_{n \kappa}+C_{p s}\right]-\left[\frac{E_{n \kappa}^{2}-M^{2}-C_{p s}\left(M+E_{n \kappa}\right)}{\eta^{2}}\right]\right)}=0,
\end{gathered}
$$

In order to calculate the wave function $G_{n \kappa}(r)$, we find the functions as,

$$
\begin{gathered}
G_{n \kappa}(r)=N_{n \kappa}\left(e^{\eta r}-1\right)^{\frac{1}{2}+\sqrt{\frac{1}{4}+\kappa[\kappa-1]}} \\
\left(e^{\eta r}\right)-\sqrt{\frac{V_{0} a}{\eta^{2}}\left[M-E_{n \kappa}+C_{p s}\right]-\left[\frac{E_{n \kappa}^{2}-M^{2}-C_{p s}\left(M+E_{n \kappa}\right)}{\eta^{2}}\right]} \\
,{ }_{2} F_{1}\left(-n, n+2 \sqrt{\frac{1}{4}+\kappa[\kappa-1]}\right.
\end{gathered}
$$

Table 1: Energies in the Spin Symmetry Limit for $\eta=$ $-0.05 \mathrm{fm}^{-1}, M=1 \mathrm{fm}^{-1}, C_{s}=5 \mathrm{fm}^{-1}, V_{0}=-0.5 \mathrm{fm}^{-1}, a=$ $-1 \mathrm{fm}^{-1}$

\begin{tabular}{|c|c|c|c|c|c|c|}
\hline$l$ & $\mathrm{n}, \kappa<0$ & $(l, j)$ & $E_{n \kappa}\left(\mathrm{fm}^{-1}\right)$ & $\mathrm{n}, \kappa>0$ & $(l, j)$ & $E_{n \kappa}\left(\mathrm{fm}^{-1}\right)$ \\
\hline 1 & $1,-2$ & $1 p_{\frac{3}{2}}$ & 0.500496102 & 1,1 & $1 p_{\frac{1}{2}}$ & 0.501270302 \\
2 & $1,-3$ & $1 d_{\frac{5}{2}}$ & 0.500546961 & 1,2 & $1 d_{\frac{3}{2}}$ & 0.501607882 \\
3 & $1,-4$ & $1 f_{\frac{7}{2}}$ & 0.500578667 & 1,3 & $1 f_{\frac{5}{2}}$ & 0.501829528 \\
4 & $1,-5$ & $1 g_{\frac{9}{2}}$ & 0.500600301 & 1,4 & $1 g_{\frac{7}{2}}$ & 0.501985253 \\
1 & $2,-2$ & $2 p_{\frac{3}{2}}$ & 0.501607882 & 2,1 & $2 p_{\frac{1}{2}}$ & 0.502512965 \\
2 & $2,-3$ & $2 d_{\frac{5}{2}}$ & 0.501829528 & 2,2 & $2 d_{\frac{3}{2}}$ & 0.503152840 \\
3 & $2,-4$ & $2 f_{\frac{7}{2}}$ & 0.501985253 & 2,3 & $2 f_{\frac{5}{2}}$ & 0.503619815 \\
4 & $2,-5$ & $2 g_{\frac{9}{2}}$ & 0.502100386 & 2,4 & $2 g_{\frac{7}{2}}$ & 0.503973169 \\
\hline
\end{tabular}

$$
\begin{gathered}
-2 \sqrt{\frac{V_{0} a}{\eta^{2}}\left[M-E_{n \kappa}+C_{p s}\right]-\left[\frac{E_{n \kappa}^{2}-M^{2}-C_{p s}\left(M+E_{n \kappa}\right)}{\eta^{2}}\right]}+1 \\
\left.2 \sqrt{\frac{1}{4}+\kappa[\kappa-1]}+1 ; 1-e^{\eta r}\right)
\end{gathered}
$$

where $N_{n \kappa}$ is the normalization constant. The upper component of the Dirac spinor can be calculated as

$$
F_{n \kappa}(r)=\frac{1}{\left(M-E_{n \kappa}+C_{p s}\right)}\left[\frac{d}{d r}-\kappa W(r)\right] G_{n \kappa}(r),
$$

where $E_{n \kappa} \neq M+C_{p s}$ and only negative energy solution is valid because negative energy spectrum is obtained in the pseudospin symmetry limit [12]. In order to show the improved accuracy of our work, we computed the energy levels for different values of the Hylleraas parameters in Table 1-4. In Tables (1) and (2) we have portrayed the energy eigenvalues for various values of the quantum numbers $n$ and $\kappa$. As we expect the energy eigenvalues in the spin symmetry limit is positive and for the pseudospin symmetry limit is negative. In Tables (3) and (4), we have reported the energy for some different values of $C_{S}$ and $C_{p s}$ respectively. We have obtained the relation between energy and potential parameter $V_{0}$ for both of the two symmetry limits in Tables (5) and (6). Fig. (1) represents the relationship between the energy and parameter of $\eta$ for pseudospin and spin symmetry limits. It is seen that if the $\eta$-parameter increases, the bound states become more bounded both for the pseudospin and spin symmetry limits.In Figs. (2), we obtain the effects of $a$-parameter on the bound states in view of the pseudospin and spin symmetry limits.We can see bound states obtained in view of spin and pseudospin symmetries become less bounded with increasing $a$.

\section{Conclusion}

In this paper, we obtained the solution of the Dirac equation for modified Hylleraas potential under the spin and pseudospin symmetry within the frame work of Alhaidariformulation using the NIkiforov-Uvarov method by approximating the less singular spin-orbit angular momentum term $\frac{1}{r}$. We obtain explicitly the energy 
Table 2: Energies in the Pseudospin Symmetry Limit for $\eta=$ $-0.05 \mathrm{fm}^{-1}, M=1 \mathrm{fm}^{-1}, C_{p s}=-5 \mathrm{fm}^{-1}, V_{0}=0.5 \mathrm{fm}^{-1}, a=$ $-1 \mathrm{fm}^{-1}$

\begin{tabular}{|c|c|c|c|c|c|c|}
\hline$l$ & $\mathrm{n}, \kappa<0$ & $(l, j)$ & $E_{n \kappa}\left(\mathrm{fm}^{-1}\right)$ & $\mathrm{n}-1, \kappa>0$ & $(l+2, j+1)$ & $E_{n \kappa}\left(\mathrm{fm}^{-1}\right)$ \\
\hline 1 & $1,-1$ & $1 s_{\frac{1}{2}}$ & -0.501270302 & 0,2 & $0 d_{\frac{3}{2}}$ & -0.500496102 \\
2 & $1,-2$ & $1 p_{\frac{3}{2}}$ & -0.501607882 & 0,3 & $0 f_{\frac{5}{2}}$ & -0.500546961 \\
3 & $1,-3$ & $1 d_{\frac{5}{2}}$ & -0.501829528 & 0,4 & $0 g_{\frac{7}{2}}$ & -0.500578667 \\
4 & $1,-4$ & $1 f_{\frac{7}{2}}$ & -0.501985253 & 0,5 & $0 h_{\frac{9}{2}}$ & -0.500600301 \\
1 & $2,-1$ & $2 s_{\frac{1}{2}}$ & -0.502512965 & 1,2 & $1 d_{\frac{3}{2}}$ & -0.501607882 \\
2 & $2,-2$ & $2 p_{\frac{3}{2}}$ & -0.503152840 & 1,3 & $1 f_{\frac{5}{2}}$ & -0.501829528 \\
3 & $2,-3$ & $2 d_{\frac{5}{2}}$ & -0.503619815 & 1,4 & $1 g_{\frac{7}{2}}$ & -0.501985253 \\
4 & $2,-4$ & $2 f_{\frac{7}{2}}$ & -0.503973169 & 1,5 & $1 h_{\frac{9}{2}}$ & -0.502100386 \\
\hline
\end{tabular}

Table 3: SS: Energies in Spin Symmetry Limit for $\eta=$ $-0.05 \mathrm{fm}^{-1}, M=1 \mathrm{fm}^{-1}, V_{0}=-0.5 \mathrm{fm}^{-1}, a=-1 \mathrm{fm}^{-1}$

\begin{tabular}{|c|c|c|c|c|}
\hline$C_{s}$ & $1 p_{\frac{3}{2}}$ & $1 d_{\frac{5}{2}}$ & $2 f_{\frac{7}{2}}$ & $2 g_{\frac{9}{2}}$ \\
\hline 3 & 0.501158302 & 0.501277129 & 0.504644008 & 0.504914058 \\
3.2 & 0.501021856 & 0.501126666 & 0.504094831 & 0.504332772 \\
3.4 & 0.500914183 & 0.501007936 & 0.503662029 & 0.503874712 \\
3.6 & 0.500827045 & 0.500911854 & 0.503312102 & 0.503504390 \\
3.8 & 0.500755079 & 0.500832502 & 0.503023298 & 0.503198770 \\
4 & 0.500694638 & 0.500765860 & 0.502780871 & 0.502942238 \\
4.2 & 0.500643157 & 0.500709098 & 0.502574471 & 0.502723836 \\
4.4 & 0.500598783 & 0.500660172 & 0.502396617 & 0.502535644 \\
4.6 & 0.500560137 & 0.500617563 & 0.502241765 & 0.502371795 \\
4.8 & 0.500526178 & 0.500580121 & 0.502105721 & 0.502227849 \\
5 & 0.500496102 & 0.500546961 & 0.501985253 & 0.502100386 \\
\hline
\end{tabular}

Table 4: PSS: Energies in Pseudospin Symmetry Limit for $\eta=$ $-0.05 \mathrm{fm}^{-1}, M=1 \mathrm{fm}^{-1}, V_{0}=0.5 \mathrm{fm}^{-1}, a=-1 \mathrm{fm}^{-1}$

\begin{tabular}{|c|c|c|c|c|}
\hline$C_{p s}$ & $1 p_{\frac{3}{2}}$ & $1 d_{\frac{5}{2}}$ & $2 f_{\frac{7}{2}}$ & $2 s_{\frac{1}{2}}$ \\
\hline-5 & -0.501607882 & -0.501829528 & -0.503973169 & -0.502512965 \\
-4.8 & -0.501705427 & -0.501940535 & -0.504214566 & -0.502665505 \\
-4.6 & -0.501815580 & -0.502065893 & -0.504487239 & -0.502837779 \\
-4.4 & -0.501940954 & -0.502208579 & -0.504797698 & -0.503033885 \\
-4.2 & -0.502084943 & -0.502372455 & -0.505154398 & -0.503259142 \\
-4 & -0.502252029 & -0.502562627 & -0.505568526 & -0.503520583 \\
-3.8 & -0.502448258 & -0.502785983 & -0.506055205 & -0.503827702 \\
-3.6 & -0.502681997 & -0.503052055 & -0.506635397 & -0.504193642 \\
-3.4 & -0.502965154 & -0.503374414 & -0.507339036 & -0.504637140 \\
-3.2 & -0.503315289 & -0.503773080 & -0.508210422 & -0.505185856 \\
-3 & -0.503759422 & -0.504278873 & -0.509318089 & -0.505882444 \\
\hline
\end{tabular}

Table 5: SS: Energies in Spin Symmetry Limit for $\eta=$ $-0.05 \mathrm{fm}^{-1}, M=1 \mathrm{fm}^{-1}, C_{s}=5 \mathrm{fm}^{-1}, a=-1 \mathrm{fm}^{-1}$

\begin{tabular}{|c|c|c|c|c|}
\hline$V_{0}$ & $1 p_{\frac{3}{2}}$ & $1 d_{\frac{5}{2}}$ & $2 f_{\frac{7}{2}}$ & $2 g_{\frac{9}{2}}$ \\
\hline-1 & 0.000434075 & 0.000478573 & 0.001736865 & 0.001837579 \\
-0.8 & 0.200456926 & 0.200503767 & 0.201828365 & 0.201934390 \\
-0.6 & 0.400482318 & 0.400531763 & 0.401930047 & 0.402041975 \\
-0.4 & 0.600510698 & 0.600563053 & 0.602043712 & 0.602162239 \\
-0.2 & 0.800542627 & 0.800598256 & 0.802171613 & 0.802297568 \\
0 & 1.000578815 & 1.000638157 & 1.002316604 & 1.002450982 \\
0.2 & 1.200620177 & 1.200683761 & 1.202482359 & 1.202626370 \\
0.4 & 1.400667907 & 1.400736386 & 1.402673690 & 1.402828823 \\
0.6 & 1.600723598 & 1.600797791 & 1.602897015 & 1.603065139 \\
0.8 & 1.800789425 & 1.800870373 & 1.803161108 & 1.803344602 \\
1 & 2.000868433 & 2.000957490 & 2.003478271 & 2.003680241 \\
\hline
\end{tabular}

Table 6: PSS: Energies in Pseudospin Symmetry Limit for $\eta=$ $-0.05 \mathrm{fm}^{-1}, M=1 \mathrm{fm}^{-1}, C_{p s}=-5 \mathrm{fm}^{-1}, a=-1 \mathrm{fm}^{-1}$

\begin{tabular}{|c|c|c|c|c|}
\hline$V_{0}$ & $1 p_{\frac{3}{2}}$ & $1 d_{\frac{5}{2}}$ & $2 f_{\frac{7}{2}}$ & $2 s_{\frac{1}{2}}$ \\
\hline-1 & -2.002816466 & -2.003205136 & -2.006969440 & -2.004404230 \\
-0.8 & -1.802559797 & -1.802912948 & -1.806332000 & -1.804002310 \\
-0.6 & -1.602346043 & -1.602669636 & -1.605801652 & -1.603667714 \\
-0.4 & -1.402165265 & -1.402463873 & -1.405353448 & -1.403384815 \\
-0.2 & -1.202010372 & -1.202287583 & -1.204969644 & -1.203142478 \\
0 & -1.001876173 & -1.002134853 & -1.004637270 & -1.002932554 \\
0.2 & -0.801758779 & -0.802001252 & -0.804346625 & -0.802748944 \\
0.4 & -0.601655218 & -0.601883396 & -0.604090305 & -0.602586987 \\
0.6 & -0.401563179 & -0.401778657 & -0.403862563 & -0.402443064 \\
0.8 & -0.201480840 & -0.201684958 & -0.203658867 & -0.202314321 \\
1 & -0.001406745 & -0.001600641 & -0.003475596 & -0.002198474 \\
\hline
\end{tabular}

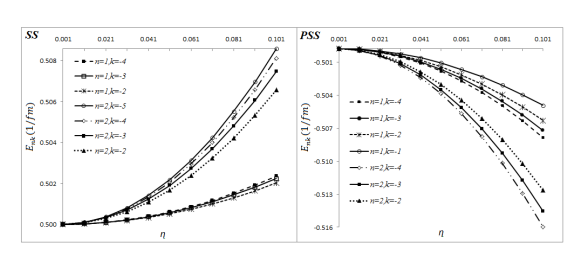

Fig. 1: SS: Energy vs. $\eta$ for Spin Symmetry Limit for $M=1 \mathrm{fm}^{-1}, C_{s}=5 \mathrm{fm}^{-1}, V_{0}=-0.5 \mathrm{fm}^{-1}, a=-1 \mathrm{fm}^{-1} \mathrm{PSS}$ : Energy vs. $\eta$ for Pseudospin Symmetry Limit for $M=$ $1 \mathrm{fm}^{-1}, C_{p s}=-5 \mathrm{fm}^{-1}, V_{0}=0.5 \mathrm{fm}^{-1}, a=-1 \mathrm{fm}^{-1}$

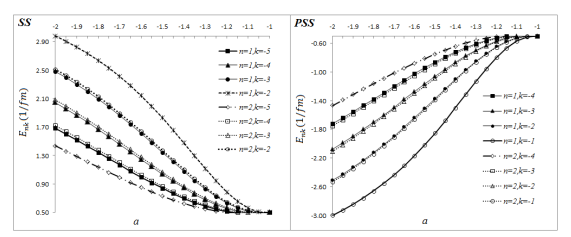

Fig. 2: SS: Energy vs. $a$ for Spin Symmetry Limit for $\eta=-0.05 \mathrm{fm}^{-1}, M=1 \mathrm{fm}^{-1}, C_{s}=5 \mathrm{fm}^{-1}, V_{0}=-0.5 \mathrm{fm}^{-1}$ PSS: Energy vs. $a$ for Pseudospin Symmetry Limit for $\eta=$ $-0.05 \mathrm{fm}^{-1}, M=1 \mathrm{fm}^{-1}, C_{p s}=-5 \mathrm{fm}^{-1}, V_{0}=0.5 \mathrm{fm}^{-1}$

eigenvalues and the corresponding wave function for both the spin and pseudospin symmetries limit. These results are extensively new and there is no available literature to compare it. However, with approximate choose on the Hylleraas parameter, the energy eigenvalues of Eq. (51) reduces to that of the Woods-Saxon [10].The wave function obtained in this work is the standardhypergeometric function [24]. Finally, as noted by Alhaidari [18] this analytic solution is valid to higher excitation levels in the spectrum than the traditional $\frac{1}{r^{2}}$ and this result is a generalized case of the one reported in Ref. [27]. 


\section{Competing interests}

Authors declare that there is no competing conflict of interest.

\section{Authors Contributions}

ANI proposed the potential and writes the manuscript. EM, OAA and SZ carried out the numerical analysis while $\mathrm{HH}$ oversees the write up and the numerical results. Finally, all the authors read and approved the final version of the manuscript.

\section{Acknowledgements}

The authors are deeply indebted to Prof.A. D. Alhaidari for his useful comments on the original version of the manuscript which has lead to the improvement of this work. A. N. Ikot dedicates the work to the memory of his late mother madam AdiahaA.A.Ndemikot. May her gentle soul rest in peace.We wish to give our sincere gratitude to the referee for a technical comment on the manuscript.

\section{References}

[1] J. N. Ginocchio, Phys. Rev.C69, 034318 (2004).

[2] J. N. Ginocchio, Phys. Rep., 414, 165 (2005).

[3] D. Troltenier, C. Bahri, J. P. Draayer, Nucl. Phys., A 586, 53 (1995).

[4] J. N. Ginocchio, Phys. Rev. Lett., 78, 436 (1997).

[5] P. R. Page, T. Goldman, J. N. Ginocchio, Phys. Rev. Lett., 86, 204 (2001).

[6] S. M. Ikhdair and R.Sever,J.Math.Phys. Doi:1063/1.3671640.

[7] S. M.Ikhdair, J. Math. Phys., 52, 52303 (2011).

[8] A.D.Alhaidari,J.Phys.A:Math.Gen., 37, 5805 (2004).

[9] S.M.Ikhdair and R.Sever,Appl.Math.Comput., 216, 911 (2010).

[10] C.Berkdemir, A.Berkdemir and R.Sever, J.Phys.A: Math.Gen., 39, 13455 (2006).

[11] M.Hamzavi,A.A.Rajabi and H.Hassanabadi,Few-Body Syst., 52, 19 (2012).

[12] A.D.Alhaidari,Phys.Lett.B, 699, 309 (2011).

[13] F.Cooper,A.Khare and U.Sukhatme,Phys.Rep., 251, 267 (1995).

[14] A.F.Nikiforov and V.B.Uvarov,Special Functions of Mathematical Physics (Birkhauser,basel, 1988).

[15] A. D.Alhaidari,Phys.Rev.A, 65, 042109 (2002).

[16] A.N.Ikot,Few-Body Syst. 53, 549-555 (2012).

[17] S.M.Ikhdair and R.Sever,J.Phys.A:Math.Theor., 44, 355301 (2011).

[18] A.D. Alhaidari, Found. Phys., 40, 1088 (2010).

[19] C.Tezcan and R.Sever,Int.J.Theor.Phys., 48, 337 (2009).

[20] M.Hamzavi,A.A.Rajabi and H.Hassanabadi,Few-Body Syst. Doi:10.1007/S00601-010-0095-7.

[21] M.Hamzavi,S.M.Ikhdair and B.I.Ita,Phys.Scr., 85, 045009 (2012).

[22] J.Meng, K.Sugawara-Tanabe, S.Yamaji and A.Arima, Phys.Rev.C59, 154, (1999).

[23] K.T.Hecht,Quantum Mechanics(Springer-Verlag,New York, 2000).

[24] I.S.Gradshteyn and I.M.Rhyzhik,Tables of Integrals,Series and Products (Elsevier,Burlington, 2007).

[25] E.A.Hylleraas,J.Chem.Phys., 3, 595 (1935). Y.P.Varshni, Rev.Mod.Phys., 29, 664 (1957).

[26] A.N.Ikot, O.A.Awoga and B.I.Ita, Few-Body Syst.2012.Doi:10.1007/s006 1-012-0434-y.

[27] A.N.Ikot,Commun.Theor.Phys., 59, 268 (2013),

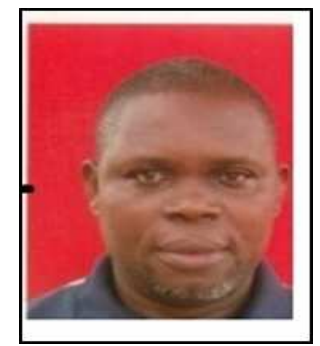

international journals.

\begin{abstract}
Akpan Ndem Ikot is a lecturer in the Dept of Physics ,University of Uyo,Nigeria. He has a Ph.D in Theoretical Physics. His research area includes Quantum Mechanics, Mathematical Physics and Computational Physics. He has more than sixty papers in
\end{abstract}

Elham Maghsoodi a lecturer in the Department of Basic Sciences, Shahrood Branch, Islamic Azad University, Shahrood, Iran . Presently, She is a Ph.D student. Her research area includes Quantum Mechanics, Mathematical Physics , Computational Physics, Condensed Matter Physics among others. She has more than seventy papers in national and international journals

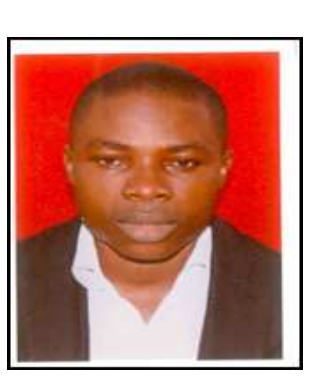

Oladunjoye

Aina Awoga a lecturer in the Dept of Physics ,University of Uyo,Nigeria. $\mathrm{He}$ is current an M.sc student in Particles Physics in Norway. His research area includes Quantum Mechanics, Mathematical Physics ,Particle Physics, Computational Physics, Condensed Matter Physics among others . He has more than twenty papers in national and international journals 


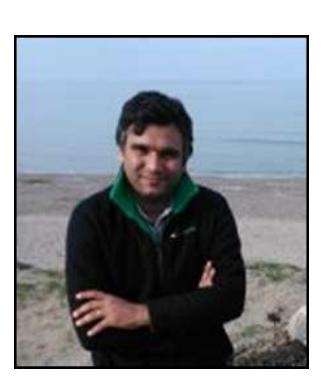

Saber Zarrinkamar is a associate Professor of Physics at university of Shahrood, Shahrood, Iran and has published 150 ISI papers in web of science journals. He has a Ph.D in Theoretical Physics. His research area includes Quantum Mechanics, Mathematical Physics , Quantum gravity, general theory of relativity Computational Physics among others. He has more than eighty papers in national and international journals to his credit. He has one hundred papers in Web of science journals to his credit.

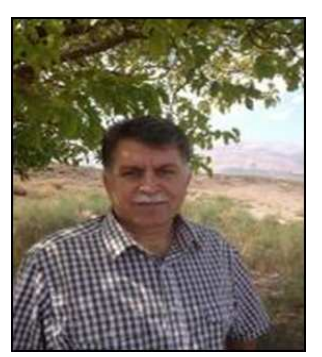

Hassan Hassanabadi is a associate Professor of Physics in the Department of Basic Sciencesat university of Shahrood, Shahrood, Iran. He has a Ph.D in Theoretical Physics. His research area includes Quantum Mechanics, Mathematical Physics , Quantum gravity, general theory of relativity Computational Physics among others. He has more than two hundred papers in international journals to his credit 